\title{
DAMPING LOSS IN A GASCADE OF FLUTTERING PHASED AEROFOILS
}

\author{
B. S. THORNTON
}

(Received 24 November 1966, revised 30 December 1966)

\begin{abstract}
Summary. A study is made of the aerodynamic damping in a cascade of oscillating aerofoils in subsonic compressible flow with the system mode described by a constant interblade phasing predicted by Lane (9). The integral downwash equation is obtained as an extension of Possio's equation for an isolated aerofoil. Procedures for a practical solution of the equation have allowed the aerodynamic reactions and damping derivatives to be evaluated with a digital computer after minimisation of the critical flutter velocity with respect to interblade phase angle. The effect of aerodynamic lag in separation conditions as a function of reduced frequency and chordwise location is compared with results without separation.
\end{abstract}

\section{Introduction}

The problem of the stalling of blades in a compressor is still an important practical problem [1]. Since axial compressors of increasingly high compression ratio have been in operation, the incidence of blade fatigue failures has increased and the idea is generally accepted that stall flutter plays a large part in these failures. Woods [2] has studied incompressible separating flow past a cascade of aerofoils and Low and Woods [3] have studied incompressible unsteady flow through a cascade when the inlet velocity is varying in magnitude. The case of an oscillating cascade in compressible flow was analysed by the author [4] and the present paper extends sections of this work which are likely to be of practical interest. It is the aim to study the subsonic compressible flow through vibrating stages of turbomachinery for which the blade height is small compared with a blade's distance from the axis of rotation such that two dimensional theory is applicable. The prediction of the air loads on an oscillating cascade blade from such an unsteady aerodynamic theory together with a knowledge of the regions where the loss of aerodynamic damping becomes significant should enable more light to be thrown on this problem. Some results from a related problem on the stability of helicopter rotor blades in slow forward motion at low inflow conditions will also be discussed.

The aerodynamic damping of an aerofoil - which is the main source of damping - is positive in flexure and torsion at low incidences and air speeds below the critical Mach number, but unstable oscillations, where the energy extracted from the air stream offsets the damping present, can occur 
as a result of phase differences between the flexural and torsional modes. This "classical" flutter is not a common form of flutter in compressor blading because of the relatively large stiffness of compressor blades, although it has received considerable attention. In turbomachinery, stall flutter is more important and in compressor cascades occurs only in flexural modes and at operating speeds considerably lower than design speeds. The oscillations with great loss of damping can be dangerous. Stall flutter is very difficult to investigate either theoretically (potential theory fails to apply) or experimentally even for a single aerofoil, although stall flutter for the single wing case where the flow separates form some fixed point on the upper surface, as with a spoiler, has been treated [5] for incompressible flow.

The loss of damping near stalling allows the blades to vibrate sporadically with high stresses under the minor random flow fluctuations of the air stream. Although a rather rigid distinction between classical and stall flutter is sometimes held, there appears to be no definite boundary between the two and some type of merging exists [5]. The exact natures of the phenomena involved in this merging are still uncertain. The present paper deals with an investigation into the classical type flutter in cascades in subsonic compressible flow and then considers the nature and effect of the phase lag between blade motion and aerodynamic force as the separation appears near the stall.

\section{The oscillation of a staggered cascade of aerofoils in subsonic compressible flow}

(i) Remarks on Earlier Work

It is necessary to consider the apparently prohibitive number of possible system modes for a fluttering compressor blade row. Lilley [6] noted from experimental observations on the flutter of a series of elastic wings in cascade that adjacent blades were approximately $\pi$ out of phase when fluttering. Reissner [7] (and others) had previously studied the aerodynamics of the single wing oscillating under the influence of tunnel walls to which this was equivalent. It should be noted that Lilley's observations indicated that for both staggered and unstaggered cascades, adjacent blades at flutter were approximately in this "antiphase" motion and that he derived the air forces and solved the cascade flutter problem assuming this motion for the unstaggered cascade only. Also previous tunnel wall interference results for the single wing apply of course, only to the unstaggered case.

Chang and $\mathrm{Chu}[8]$ studied the oscillation of an unstaggered two dimensional cascade at small incidence under the assumption that all blades oscillate in phase with one another. This allowed a solution by mapping techniques similar to those of Theodorsen for the flutter of a single wing. 
This assumption of oscillations being in phase with one another, has, however, been shown to be the least conservative one possible [9] with respect to the prediction of critical flutter velocity, and not of great practical importance. The critical cascade flutter velocities on such a theory exceed those for a single isolated blade which is known to be opposite to the actual situation in practice.

The case of blades with phasing zero, $\pi / 2$ and $\pi$ has been studied by Mendelson and Carroll [10] but other phasings were very difficult to investigate and were not attempted. Söhngen [11] has also studied a case of a cascade of aerofoils oscillating in incompressible flow with amplitudes and phasings which need not be equal. A significant advance in the study of the actual type of system modes to be expected for compressor blades in flutter has been made by Lane [9] who investigated the dynamics of a very general multi-blade system from first principles with the idea of predicting, or at least restricting, in advance, the form of the system mode which exists at flutter. Lane proved that a system of identical blades, identically supported and equally spaced about a common rotor will flutter in a system mode, or at worst, in a linear combination of modes, in which all blades oscillate with identical blade modes and in which each blade leads or lags its neighbour by a common phase angle; the value of this angle constitutes one portion of the solution of the flutter problem for a particular configuration. The existence and uniqueness of these extremely simple system flutter modes eliminate problems associated with the otherwise prohibitive number of possible system modes for the blades. Lane's work can be interpreted as reducing by a factor of $n$ the number of degrees of freedom necessary to analyse an $n$-bladed system. The proof holds for any type of flow and even near the onset of stalling (separating and reattaching boundary layer) - so long as a linear analysis is permissible at the flutter inception point. Another result is that the "antiphase" motion observed by Lilley is to be expected for the unstaggered cascade but not necessarily for other configurations.

The actual physical system to be studied is approximated to by a two-dimensional linear cascade model shown in Figure 1 and the system mode shape established by Lane is used. For a linear cascade as shown and also in practice for a compressor rotor with many blades, the phase angle $\sigma$, between successive blades in Lane's analysis is assumed to be continuously variable and later, when the flutter velocity has been determined in terms of this angle, a minimisation of this flutter velocity with respect to $\sigma$ is done - to determine the critical flutter velocity - assuming $\sigma$ to be continuously variable. The true practical value of $\sigma$ in an actual compressor rotor with a large, but finite, number of blades will be the value closest to the determined value chosen from a discrete set of admissible $\sigma$ values as explained in Lane's paper [9]. 

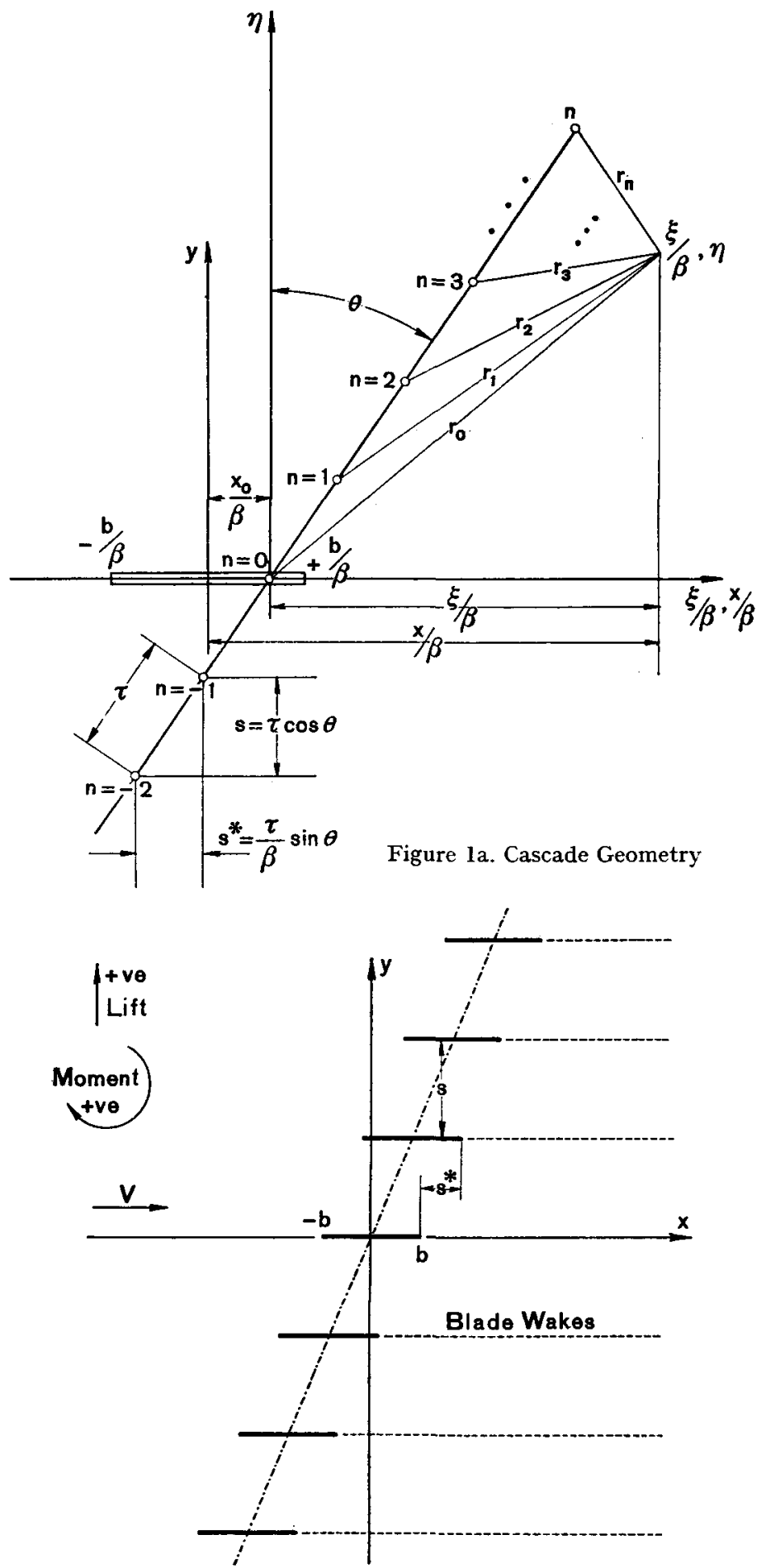

Figure 1b. Cascade of Doublets in Prandt1-Glauert Space 
Initial angle of attack of the blades is assumed to be small or zero and it is assumed that the oscillatory disturbances are sufficiently small for linearised theory to apply. The relation between a three dimensional solution and the present "strip" theory (cascade) approximation for blade rows having a large number of blades has been investigated [12]. Strip theory as herein used has been shown to correspond to the first term of a series development of the flow variables in inverse powers of the blade number. Analytical expressions for the three-dimensional corrections to strip theory have been evaluated and are proportional to $N^{-\frac{2}{3}}$ where $N$ is the number of blades in the blade row studied - and consequently are neglected in the present analysis in comparison with the nature of the other assumptions made.

The method used for the work described in the following section extends the isolated aerofoil theory of Possio [13] to the case of a cascade of aerofoils of chord $c=2 b$, stagger angle $\theta$, and oscillating with an interblade phase angle $\sigma$ determined as previously described, with frequency $\omega$ in a compressible flow of free stream velocity $V$ and free stream density $\rho_{\infty}$.

\section{(ii) The Downwash Equation for a Staggered Cascade of Aerofoils}

The physical system studied and relevant notation is shown in Figures $\mathrm{la}, \mathrm{b}$ where the phase difference between successive blades is $\sigma$. Proceeding along the lines used by Possio, the expression for the downwash per unit chord, $\bar{W}^{\prime}$, is obtained as [4]:

$$
\begin{aligned}
\bar{W}^{\prime}\left(x_{0}, x-x_{0}, \eta\right) \\
=-\frac{A\left(x_{0}\right) e^{-i \omega\left(x-x_{0}\right) / v}}{V} \lim _{\eta \rightarrow 0} \int_{-\infty}^{x-x_{0}} e^{i \omega \xi / V \beta^{2}} \sum_{n=-\infty}^{\infty} e^{2 i \pi R n} \frac{\partial^{2}}{\partial \eta^{2}} H_{0}^{(2)}\left(r_{n}^{*}\right) d \xi
\end{aligned}
$$

where

$$
R=\frac{\sigma}{\pi}-\frac{\omega M \tau \sin \theta}{2 \pi a \beta^{2}}
$$

and $r_{n}^{*}=r_{n} \omega / a, a$ being the local speed of sound. Expressing the doublet strength distribution $\bar{A}\left(x_{0}\right)$ as a function of $\Delta \bar{p}\left(x_{0}\right)$, the local lift distribution, [4],

$$
\bar{A}\left(x_{0}\right)=\frac{i \Delta \bar{p}\left(x_{0}\right)}{4 \rho_{\infty} \beta}
$$

where $\beta=\sqrt{1-M^{2}}, M$ being the Mach number, we obtain from equation (1) the total downwash at any point $(x, 0)$ in the plane of the zeroth aerofoil by integrating over $x_{0}$ from $-b$ to $+b$. This is given by 


$$
\begin{array}{r}
\bar{W}(x)=\frac{-i}{4 \rho_{\infty} V \beta} \int_{-b}^{b} \Delta \bar{p}\left(x_{0}\right) e^{-i \omega\left(x-x_{0}\right) / V} \lim _{\eta \rightarrow 0} \int_{-\infty}^{x-x_{0}} e^{i \omega \xi / V \beta^{2}} \\
\cdot \sum_{n=-\infty}^{\infty} e^{2 i \pi R n} \frac{\partial^{2}}{\partial \eta^{2}} H_{0}^{(2)}\left(r_{n}^{*}\right) d \xi d x_{0}
\end{array}
$$

which relates the known downwash at $x$ (as given by the oscillatory mode) to the unknown lift distribution on an aerofoil in the cascade. Equation (2) can be written in a simpler form:

$$
\bar{W}(x)=\frac{-i}{4 \rho_{\infty} V \beta} \int_{-b}^{b} \Delta \bar{p}\left(x_{0}\right)\left[K_{0}\left(M, x-x_{0}\right)+K_{1}\left(M, x-x_{0}, \tau\right)\right] d x_{0}
$$

where the kernels of the integral are given by

$$
\begin{aligned}
K_{0}= & e^{-i \omega\left(x-x_{0}\right) / \nabla} \lim _{\eta \rightarrow 0} \int_{-\infty}^{x-x_{0}} e^{i \omega \xi / V \beta^{2}} \frac{\partial^{2}}{\partial \eta^{2}} H_{0}^{(2)}\left(\frac{\omega}{a \beta^{2}} \sqrt{ }\left(\xi^{2}+\beta^{2} \eta^{2}\right)\right) d \xi \\
K_{1}= & e^{-i \omega\left(x-x_{0}\right) / V} \lim _{\eta \rightarrow 0} \int_{-\infty}^{x-x_{0}} e^{i \omega \xi / V \beta^{2}} \\
& \sum_{n=-\infty}^{\infty} 0 e^{2 i \pi R n} \frac{\partial^{2}}{\partial \eta^{2}} H_{0}^{(2)}\left(\frac{\omega}{a \beta^{2}} \sqrt{(\xi-n \tau \sin \theta)^{2}+\beta^{2}(\eta-n \tau \cos \theta)^{2}}\right) d \xi
\end{aligned}
$$

and where the argument of the Hankel function, $r_{n}^{*}$, has been replaced by $\omega / a \cdot r(\xi, \eta, n, \tau, \theta, \beta)$. In equation (5) the summation is carried out over all positive and negative values of $n$ excluding $n=0$. The symbol

$$
\sum_{n=-\infty}^{\infty}=\sum_{n=-\infty}^{-1}+\sum_{n=1}^{\infty}
$$

denotes this operation. It is noted that $K_{0}$ in equation (4) is the kernel of Possio's integral equation for an isolated aerofoil in compressible flow [13], and that the kernel $K_{1}$ of equation (5) represents the correction to the isolated aerofoil results for the aerofoils in cascade.

Equation (3) may be rewritten in a more useful form

$$
\begin{aligned}
\bar{W}(x) & =\frac{-i}{4 \rho_{\infty} V \beta} \int_{-b}^{b} \Delta \bar{p}\left(x_{0}\right) K_{0}\left(M, x-x_{0}\right) d x_{0} \\
& -\frac{i \omega e^{i \omega M\left(x-x_{0}\right) / a \beta^{2}}}{4 \rho_{\infty} a V \beta^{3}} \int_{-b}^{b} \Delta \bar{p}\left(x_{0}\right)\left[\frac{i}{M} S_{1}+S_{2}+\frac{\omega\left(1-M^{2} \beta^{2}\right)}{a M^{2} \beta^{2}} e^{-i \omega\left(x-x_{0}\right) / V \beta^{2}} S_{3}\right] d x_{0}
\end{aligned}
$$

where the series in the second integrand are given by

$$
S_{1}=\sum_{n=-\infty}^{\infty} e^{2 i \pi R n} H_{0}^{(2)}\left(\frac{\omega}{a \beta^{2}} \sqrt{\left(x-x_{0}-n \tau \sin \theta\right)^{2}+(\beta n \tau \cos \theta)^{2}}\right)
$$




$$
\begin{aligned}
& S_{2}= \sum_{n=-\infty}^{\infty} 0 \frac{e^{2 i \pi R n}\left(x-x_{0}-n \tau \sin \theta\right)}{\sqrt{\left(x-x_{0}-n \tau \sin \theta\right)^{2}+(\beta n \tau \cos \theta)^{2}}} \\
& H_{1}^{(2)}\left(\frac{\omega}{a \beta^{2}} \sqrt{\left(x-x_{0}-n \tau \sin \theta\right)^{2}+(\beta n \tau \cos \theta)^{2}}\right)
\end{aligned}
$$

(9) $S_{0}=\sum_{n=-\infty}^{\infty} \circ e^{2 i \pi R n} \int_{-\infty}^{x-x_{0}} e^{i \omega \xi / V \beta^{2}} H_{0}^{(2)}\left(\frac{\omega}{a \beta^{2}} \sqrt{(\xi-n \tau \sin \theta)^{2}+(\beta n \tau \cos \theta)^{2}}\right) d \xi$.

Equation (6) represents the complete solution of the unsteady aerodynamic problem for aerofoils in cascade having arbitrary stagger and oscillating in compressible flow and is analogous to Possio's solution for the isolated aerofoil.

Unfortunately, this solution has attendant difficulties since there is no known closed solution for the series of equations (7), (8) and (9) for the arbitrary cascade. However, for $\theta=0$ (an unstaggered cascade) some progress can be made as will be shown.

Another difficulty is that the above series $S_{1}, S_{2}$ and $S_{3}$ are divergent for certain values of the geometric and flow parameters so that the kernel of the integral equation is then unbounded. This leads to a phenomenon which is analogous to the acoustical resonance of an isolated aerofoil in a wind tunnel.

Before discussing the more practical presentation of the above series leading to the determination of aerodynamic damping coefficients in an unstaggered cascade, some comment on the convergence of these series is of interest. This is advisable because for the particular values of the geometric and flow parameters, which it will be shown, make the series divergent; any numerical calculation using these series would fail to give sufficient accuracy in the neighbourhood of these values.

The physical aspects and significance can readily be interpreted by the following considerations:

Let the blades oscillate at frequency $\omega \mathrm{rad} / \mathrm{sec}$ and with an interblade phase angle $\sigma$, where positive $\sigma$ implies that the reference blade leads the lower adjacent blade by that amount. For reinforcement of the signal, the propagation time for the pressure signal to traverse the distance between blades along the stagger line must equal the time lag of the oscillatory motion of adjacent blades (which is proportional to the interblade phase angle). The time lag for the lower adjacent aerofoil is

$$
t_{\text {lag, lower }}=\sigma / \omega,(\sigma+2 \pi) / \omega, \cdots,(\sigma+2 \pi v) / \omega
$$

and for the upper

$$
t_{\text {lag, upper }}=(2 \pi-\sigma) / \omega,(4 \pi-\sigma) / \omega, \cdots,(2 \pi \nu-\sigma) / \omega
$$


or, in general

$$
t_{\text {lag }}=(2 \pi \nu \pm \sigma) / \omega
$$

where for $+\sigma, \nu=0,1,2, \cdots$ are the permissible values and for $-\sigma$ $v=1,2,3, \cdots$ are the permissible values.

The time required for a pressure signal to propagate between adjacent aerofoils is

$$
t_{\text {prop }}=\tau / V_{\text {prop }}
$$

where $V_{\text {prop }}$ is the propagation velocity along the stagger line (see Figure 2): from the law of cosines

$$
\begin{aligned}
& V_{\mathrm{prop}, l}=-V \sin \theta+\sqrt{V^{2} \sin ^{2} \theta+\left(\alpha^{2}-V^{2}\right)} \\
& V_{\mathrm{prop}, u}=V \sin \theta+\sqrt{V^{2} \sin ^{2} \theta+\left(\alpha^{2}-V^{2}\right)}
\end{aligned}
$$

and, in general,

$$
V_{\text {prop }}= \pm V \sin \theta+\sqrt{V^{2} \sin ^{2} \theta+\left(\alpha^{2}-V^{2}\right)},
$$

$a$ being the local speed of sound. Now set $t_{\text {lag }}=t_{\text {prop }}$, make the substitutions $M=V / a, k_{a}=\omega b / a=\omega c / 2 a$ and the result is

$$
\frac{2 \tau}{c} k_{a} \cos \theta=\frac{\pi\left(1-M^{2}\right)(2 v \pm \sigma / \pi)}{\sqrt{1-M^{2}+\tan ^{2} \theta \pm M \tan \theta}} .
$$

The series of equations (7), (8) and (9) are divergent for values of the geometric and flow parameters which satisfy equation (10), and at these values the lift distribution must vanish to satisfy physical reasoning. If such a condition is actually encountered by a blade system the aerodynamic forces

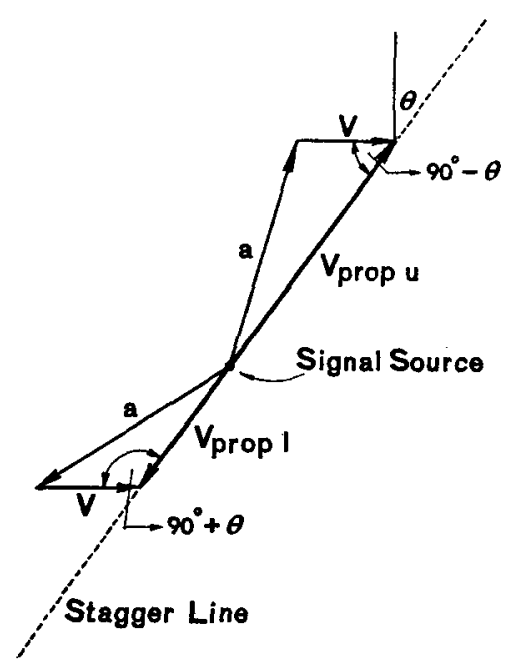

Figure 2. Signal Propagation. Signal propagated outwards at speed of sound, a, carried downstream by free stream velocity, $V$. 
must also vanish. If, under such unfortunate conditions, and external oscillatory energy source imparts energy to the blades at a frequency equal to the natural blade frequency, there is no aerodynamic dissipation of the energy, consequently the amplitude of the blade vibration is then limited only by the very small structural damping of the system. Fortunately, the radial variations in both incident flow and cascade geometry for an actual compressor with tapered twisted blades would probably make it impossible for a large radial extent of blades to be simultaneously at this resonant condition - but the situation can still be dangerous. The stability of the non linear differential-difference equation governing blade bending and small torsional oscillations near the onset of this condition, in the presence of disturbances from upstream wakes, has been analysed by digital computer [14] and the program is available from the author (Latest report currently in the press, J. Roy. Aero. Soc.).

The existence of the resonant condition described has also been arrived at by Lane and Friedman [20] by studying the poles of the integral they obtain for the downwash velocity in terms of the Fourier transform of the pressure distribution.

The derivations of the resonant condition, both in the present report and in Lane and Friedman's paper can be usefully interpreted by the above derivation which shows the important physical aspects more clearly, than a formal and lengthy derivation of the conditions for convergence (which is, however, available [4]).

\section{The lift, moment and damping derivatives}

(i) Practical Evaluation of the Series $S_{1}, S_{2}, S_{3}$

Before dealing with the practical evaluation of damping derivatives near the stall which requires the introduction of aerodynamic lags, it will be necessary to put the series $S_{1}, S_{2}$, and $S_{3}$ into forms suitable for numerical computation.

First consider $S_{1}$. For zero stagger

$$
S_{1}=\sum_{n=-\infty}^{\infty} e^{i 2 \pi R n} H_{0}^{(2)}\left(2 \pi z \sqrt{\left.n^{2}+\varepsilon^{2}\right)}\right.
$$

where $z=\omega \tau / 2 \pi a \beta$ and $\varepsilon=\left(x-x_{0}\right) / \beta \tau$. We now use a method arising from the theory of Bessel Functions. It is known [15], [16] that

$$
\begin{aligned}
\int_{0}^{\infty} J_{\mu}(x t) & \frac{K_{\nu}\left(\varepsilon \sqrt{x^{2}-z^{2}}\right)}{\left(x^{2}-z^{2}\right)^{\nu / 2}} x^{\mu+1} d x \\
= & \frac{\pi}{2} e^{i \pi\left(\nu-\mu-\frac{1}{2}\right)} \frac{t^{\mu}}{\varepsilon^{\nu}}\left(\frac{\sqrt{\varepsilon^{2}+t^{2}}}{z}\right)^{\nu-\mu-1} H_{\nu-\mu-1}^{(2)}\left(z \sqrt{\varepsilon^{2}+t^{2}}\right)
\end{aligned}
$$


valid for real $(v)<1, z>0$. Putting $\mu=-\frac{1}{2}, v=\frac{1}{2}$ results in

$$
\frac{1}{\sqrt{2 \pi}} \int_{-\infty}^{\infty} \frac{e^{-\varepsilon \sqrt{x^{2}-z^{2}}}}{\sqrt{x^{2}-z^{2}}} e^{i t x} d x=-\sqrt{\frac{\pi}{2}} H_{0}^{(2)}\left(z \sqrt{t^{2}+\varepsilon^{2}}\right) .
$$

Thus, the Fourier transform of

$$
\frac{e^{-\varepsilon \sqrt{x^{2}-z^{2}}}}{\sqrt{x^{2}-z^{2}}} \text { is } \sqrt{\frac{\pi}{2}} e^{i \pi / 2} H_{0}^{(2)}\left(z \sqrt{t^{2}+\varepsilon^{2}}\right) .
$$

Therefore, by the reciprocal property of the Fourier transform:

$$
\int_{-\infty}^{\infty} H_{0}^{(2)}\left(z \sqrt{t^{2}+\varepsilon^{2}}\right) e^{i t x} d x=-\frac{2 i e^{-\varepsilon \sqrt{x^{2}-z^{2}}}}{\sqrt{x^{2}-z^{2}}} .
$$

Now consider

$\frac{1}{\sqrt{2 \pi}} \int_{-\infty}^{\infty} e^{-i x t} e^{i 2 \pi R t} H_{0}^{(2)}\left(z \sqrt{t^{2}+\varepsilon^{2}}\right) d t=\frac{1}{\sqrt{2 \pi}}-2 i \frac{\exp \left(-\varepsilon \sqrt{\left.(-x+2 \pi R)^{2}-z^{2}\right)}\right.}{\sqrt{\left.(-x+2 \pi R)^{2}-z^{2}\right)}}$

from equation (13). Therefore the Fourier transform of

is

$$
g(t)\left(=e^{i 2 \pi R t} H_{0}^{2}\left(z \sqrt{t^{2}+\varepsilon^{2}}\right)\right)
$$

$$
f(x)=-i \frac{\sqrt{2 / \pi} \exp \left(-\varepsilon \sqrt{\left.(x-2 \pi R)^{2}-z^{2}\right)}\right.}{\sqrt{(x-2 \pi R)^{2}-z^{2}}} .
$$

Now the Poisson summation formula [17] states that

$$
\sum_{n=-\infty}^{\infty} f(2 \pi n)=\frac{1}{2 \pi} \sum_{n=-\infty}^{\infty} g(n)
$$

where $g(x)$ is the Fourier transform of $f(x)$. Replacing $z$ by $2 \pi z$ for convenience we have from equation (14),

$$
\frac{1}{2 \pi} \sum_{n=-\infty}^{\infty} e^{i 2 \pi R n} H_{0}^{(2)}\left(2 \pi z \sqrt{n^{2}+\varepsilon^{2}}\right)=-\frac{i}{2 \pi} \sqrt{\frac{2}{\pi}} \sum_{n=-\infty}^{\infty} \frac{\exp \left(-2 \pi \varepsilon \sqrt{\left.(n-R)^{2}-z^{2}\right)}\right.}{\sqrt{(n-R)^{2}-z^{2}}} .
$$

Therefore from equation (11),

$$
S_{1}=--i \sqrt{\frac{2}{\pi}} \sum_{n=-\infty}^{\infty} \frac{\exp \left[-2 \pi\left(\overline{x-x_{0}} / \beta \tau\right) \sqrt{(n-R)^{2}-(\omega \tau / a \beta)^{2}}\right]}{\sqrt{(n-R)^{2}-(\omega \tau / a \beta)}}-H_{0}^{(2)}\left(\frac{\omega \tau \varepsilon}{a \beta}\right) .
$$

For $S_{2}$, following exactly the same procedure as before after putting (in this case) $\mu=\nu=-\frac{1}{2}$ in the relation (12), we get 


$$
\begin{aligned}
S_{2}=- & 2 i \frac{\sqrt{2 \pi} a}{\omega \tau^{2}}\left(x-x_{0}\right) \\
& \sum_{n=-\infty}^{\infty} \circ \exp \left[-2 \pi\left(\frac{x-x_{0}}{\beta \tau}\right) \sqrt{(n-R)^{2}-\left(\frac{\omega \tau}{a \beta}\right)^{2}}\right]-H_{1}^{(2)}\left(\frac{\omega \tau \varepsilon}{a \beta}\right) .
\end{aligned}
$$

For $S_{3}$,

$$
S_{3}=\sum_{n=-\infty}^{\infty} e^{2 \pi i R n} \int_{-\infty}^{x-x_{0}} e^{i \omega \xi / V \beta^{2}} H_{0}^{(2)}\left(\frac{\omega}{a \beta^{2}} \sqrt{\xi^{2}+(\beta n \tau)^{2}}\right) d \xi
$$

This type of expression with $e^{2 \pi i R n}$ replaced by $(-1)^{n}$ occurs in the case of a single wing oscillating in subsonic compressible flow in a wind tunnel [18] and in evaluating that series it was assumed that the tunnel height was large compared with the wing chord and certain simplifications resulted. In the present case of compressor blades in cascade this assumption is not valid and we proceed as follows.

Write the expression (17) as

$$
\begin{aligned}
S_{3}= & \int_{0}^{\infty} e^{-i \omega \xi / V \beta^{2}}\left[\sum_{n=-\infty}^{\infty} \circ e^{2 \pi i R^{n}} H_{0}^{(2)}\left(\frac{\omega}{a \beta^{2}} \sqrt{\xi^{2}-(\beta n \tau)^{2}}\right)\right] d \xi \\
& +\sum_{n=-\infty}^{\infty} \circ e^{2 \pi i R n} \int_{0}^{x-x_{0}} e^{i \omega \xi / V \beta^{2}} H_{0}^{(2)}\left(\frac{\omega}{a \beta^{2}} \sqrt{\xi^{2}-(\beta n \tau)^{2}}\right) d \xi .
\end{aligned}
$$

The first integral on the right hand side can be found in a convenient series form since the expression in square brackets is $S_{1}$ - which has been evaluated. The integration can then be done term by term after multiplying by $e^{-i \omega \xi / V \beta^{2}}$. (The terms involving $\xi$ in $S_{1}$ are of the form $e^{-(1) \xi}$ ). The second integral on the right hand side is best done by numerical means [19].

\section{(ii) Evaluation of the Lift, Moment \& Damping Derivatives}

Having determined $S_{1}, S_{2}$ and $S_{3}$ the equation (6) can then be solved for $\Delta \bar{p}\left(x_{0}\right)$ in a similar way to the manner in which the Possio equation for the isolated wing can be treated by the method of collocation. In our case we take

$$
\frac{\Delta \bar{p}\left(x_{0}\right)}{\rho_{\infty} V^{2}}=A_{0} \cot \Theta / 2+\sum_{n=1}^{\infty} A_{n} \sin n \Theta
$$

with $x_{0}=-b \cos \Theta$. Note that the $\cot \Theta / 2$ term gives the proper leading edge singularity and vanishing trailing edge value of pressure jump in accordance with one form of the Kutta condition.

Lane and Friedman [20] showed that if the pressure jump is taken in the above form, then the lift and nose-up moment (about mid-chord) amplitudes $L, \bar{M}$ are given by 


$$
L / \rho_{\infty} V^{2} b=-\pi\left(A_{0}+A_{1} / 2\right)
$$

and

$$
\bar{M} / \rho_{\infty} V^{2} b=-\pi / 2\left(A_{0}+A_{2} / 2\right) .
$$

To find the coefficients $A_{0}, A_{1}$ and $A_{2}$ the expansion (19) is substituted into equation (6) and the value of $W(x)$, formed from the known aerofoil motion at a number of chord stations or "control points", is substituted on the left hand side of the equation (6). The integral is then evaluated in terms of the coefficients $A_{0}, A_{1} \cdots$ at each of these control points. This gives a number $(N)$ of simultaneous algebraic equations for a limited number $(N)$ of the $A_{n}$. These are then solved to find the $A_{n}$.

Runyan and Woolston [18] found that for a single wing in a tunnel which is equivalent to a cascade with an interblade phase angle of $\pi$ that the first three terms of the series for the pressure jump and three control points at the $\frac{1}{4}, \frac{1}{2}$ and $\frac{3}{4}$ chord positions gave satisfactory results and a larger number of control points did not improve the accuracy to a significant degree. However, for high values of the reduced frequency parameter the use of additional control points might be necessary.

For the calculations done in connection with the present report three control points $\frac{1}{4} c, \frac{1}{2} c, \frac{3}{4} c$ and the first three terms of the pressure expansion were used.

Once $A_{0}, A_{1}$ and $A_{2}$ are known the lift and moment amplitudes are known from equations (20) and (21). However, it is the damping derivatives which are of greater interest since in practical problems of compressor blade flutter it is the sign of the damping in a particular degree of freedom which determines whether the motion in that degree of freedom is damped or not by the aerodynamic forces.

Since experimental data on compressors indicates that the important type of vibration occurring is one of bending we consider the cascade of aerofoils only in bending modes not in torsion modes. However, a small degree of torsion can also exist in practice but this only becomes important with respect to stability of vibrations when the separation region is entered $[14]$.

The "air load" coefficients $l_{12}$ and $m_{12}$ are defined in terms of the lift $L$ and moment $M$ by

$$
\begin{gathered}
L / \rho_{\infty} c V^{2} e^{i \omega t}=l_{12} \cdot h_{0} / c \\
M / \rho_{\infty} c^{2} V^{2} e^{i \omega t}=m_{12} \cdot h_{0} / c
\end{gathered}
$$

where $h_{\mathbf{0}}$ is the amplitude of blade oscillation in bending. The coefficients $l_{12}$ and $m_{12}$ are in general complex so that 


$$
\begin{aligned}
l_{12} & =l_{1}+i l_{2} \\
m_{12} & =m_{1}+i m_{2}
\end{aligned}
$$

$l_{1}, l_{2}, m_{1}, m_{2}$ being real numbers. (The quantities $l_{1}$ and $m_{1}$ are termed stiffness derivatives while $l_{2}$ and $m_{2}$ are termed damping derivatives). The real parts $l_{1}, m_{1}$ are in phase with the displacement and can do no work, whereas the imaginary parts $l_{2}, m_{2}$ are in phase with the velocity and correspond to damping components and can do work.

From equations (20)-(23) it is seen that the aerodynamic derivatives $l_{1}, l_{2}, m_{1}, m_{2}$ are found once $A_{0}, A_{1}$ and $A_{2}$ are known.

\section{The damping derivatives near the stall}

\section{(i) The Aerodynamic Lag}

Close to the stall region classical flutter theory is inadequate and results in incorrect predictions. It is well known that for an aerofoil oscillating near the stall region, the aerodynamic forces acting on the aerofoil tend to lag the motion, producing a hysteresis curve and enabling the aerofoil to absorb sufficient energy from the air stream to maintain its oscillations. This lag is most important; if it were known for the compressor blade cascade it could be incorporated as an "angle of aerodynamic lag".

Various semi-empirical attempts have been made to predict the dynamic behaviour of aerofoils in or near the stall region or separated flow. Most of these methods start with steady flow experimental characteristics or with non-steady potential flow characteristics and introduce such concepts as modifying the effective camber, relaxing the Kutta condition, introducing an adjustable time lag or other arbitrary assumptions. Although such devices may lead to successful treatment in specific cases and over limited ranges of the variables, none as yet may be considered sufficiently general solutions of the complex problems involved. However, after consideration of experimental information available, it was decided that the most profitable line of approach in studying the region near the stall for compressor cascades is that of aerodynamic lag angle, $\varphi$.

Experimental information previously communicated ${ }^{\mathbf{1}}$ to the author from United Aircraft Corporation [4] indicates that the lag in the flow separation region is a function of reduced frequency $k=\omega b / V$ and of chordwise location, as included in Tables I, II. It also appears that the lag does not seem to vary much with the mean angle of attack for a fixed value of the reduced frequency. The experimental information was obtained from

1 F. O. Carta, private communication. 
TABLE I

Damping Derivative $l_{2}$ $\sigma=200^{\circ}$, zero stagger angle, Aerofoil section N.A.C.A. $\langle 2.1\rangle 405$

\begin{tabular}{|c|c|c|c|c|c|c|c|c|}
\hline \multirow{3}{*}{$\begin{array}{l}\text { Reduced } \\
\text { frequency } \\
k\end{array}$} & \multirow{2}{*}{\multicolumn{3}{|c|}{$\begin{array}{c}\text { Aerodynamic phase } \\
\operatorname{Lag} \varphi\end{array}$}} & $\begin{array}{l}\text { Curves } \\
\text { la, b }\end{array}$ & $\begin{array}{l}\text { Curves } \\
2 \mathrm{a}, \mathrm{b}\end{array}$ & $\begin{array}{l}\text { Curves } \\
3 \mathrm{a}, \mathrm{b}\end{array}$ & $\begin{array}{c}\text { Curve } \\
4\end{array}$ & $\begin{array}{c}\text { Curve } \\
5\end{array}$ \\
\hline & & & & \multirow{2}{*}{$\begin{array}{l}l_{\mathrm{2}} \text { for } \\
s / c=0.75 \\
M=0.5\end{array}$} & \multirow{2}{*}{$\begin{array}{l}l_{2} \text { for } \\
s / c=1.00 \\
M=0.5\end{array}$} & \multirow{2}{*}{$\begin{array}{l}l_{2} \text { for } \\
s / c=1.50 \\
M=0.5\end{array}$} & \multirow{2}{*}{$\begin{array}{l}l_{2} \text { for } \\
\text { isolated } \\
\text { wing, } \\
\text { separation } \\
\text { from } \\
\text { leading } \\
\text { edge } \\
M=0\end{array}$} & \multirow{2}{*}{$\begin{array}{l}l_{2} \text { for } \\
\text { isolated } \\
\text { wing, } \\
\text { separation } \\
60 \% \\
\text { back from } \\
\text { leading } \\
\text { edge, } \\
M=0\end{array}$} \\
\hline & $\frac{1}{4} c$ & $\frac{1}{2} c$ & $\frac{3}{4} c$ & & & & & \\
\hline 0.02 & $\begin{array}{c}3^{\circ} 30^{\prime} \\
0\end{array}$ & $\begin{array}{l}3^{\circ} \\
0\end{array}$ & $\begin{array}{c}2^{\circ} 30^{\prime} \\
0\end{array}$ & $\begin{array}{l}-0.0652 \\
-0.0652\end{array}$ & $\begin{array}{l}-0.0894 \\
-0.0895\end{array}$ & $\begin{array}{l}-0.2196 \\
-0.2196\end{array}$ & -0.0291 & -0.0352 \\
\hline 0.10 & $\begin{array}{c}17^{\circ} \\
0\end{array}$ & $\begin{array}{c}16^{\circ} 30^{\prime} \\
0\end{array}$ & $\begin{array}{c}13^{\circ} \\
0\end{array}$ & $\begin{array}{l}-0.2721 \\
-0.2804\end{array}$ & $\begin{array}{l}-0.4018 \\
-0.3920\end{array}$ & $\begin{array}{l}-0.6520 \\
-0.7104\end{array}$ & -0.1552 & -0.1997 \\
\hline 0.20 & $\begin{array}{c}35^{\circ} \\
0\end{array}$ & $\begin{array}{c}32^{\circ} 30^{\prime} \\
0\end{array}$ & $\begin{array}{c}25^{\circ} 30^{\prime} \\
0\end{array}$ & $\begin{array}{l}-0.4801 \\
-0.4910\end{array}$ & $\begin{array}{l}-0.6425 \\
-0.6426\end{array}$ & $\begin{array}{l}-1.0106 \\
-1.0853\end{array}$ & -0.3040 & -0.3927 \\
\hline 0.30 & $\begin{array}{c}52^{\circ} 30^{\prime} \\
\quad 0\end{array}$ & $\begin{array}{c}49^{\circ} \\
0\end{array}$ & $\begin{array}{c}38^{\circ} 30^{\prime} \\
0\end{array}$ & $\begin{array}{l}-0.6098 \\
-0.6688\end{array}$ & $\begin{array}{l}-0.7273 \\
-0.8535\end{array}$ & $\begin{array}{l}-1.1592 \\
-1.4103\end{array}$ & -0.4491 & -0.5904 \\
\hline 0.40 & $\begin{array}{c}10^{\circ} \\
0\end{array}$ & $\begin{array}{c}65^{\circ} 30^{\prime} \\
0\end{array}$ & $\begin{array}{c}51^{\circ} \\
0\end{array}$ & $\begin{array}{l}-0.4214 \\
-0.8153\end{array}$ & $\begin{array}{l}-0.5256 \\
-1.0831\end{array}$ & $\begin{array}{l}-1.1330 \\
-1.6561\end{array}$ & -0.6104 & -0.7910 \\
\hline 0.50 & $\begin{array}{c}95^{\circ} \\
0\end{array}$ & $\begin{array}{c}81^{\circ} 30^{\prime} \\
0\end{array}$ & $\begin{array}{c}63^{\circ} 30^{\prime} \\
0\end{array}$ & $\begin{array}{l}-0.2143 \\
-0.9558\end{array}$ & $\begin{array}{r}-0.3930 \\
-1.3572\end{array}$ & $\begin{array}{l}-1.0147 \\
-2.1774\end{array}$ & -0.7608 & -0.9898 \\
\hline
\end{tabular}

Practical $\varphi$ values not available beyond $k=0.50$.

high speed movie interferometer studies in which the motion of the boundary layer was correlated with the instantaneous pressure and the motion of the aerofoil.

Although the concept of an aerodynamic phase lag angle is incorporated it is worth noting that it is not definitely known yet whether the lag is primarily an unsteady boundary layer effect or whether it is a function of the quasi-steady lift curve.

\section{(ii) Numerical Results for Damping Derivatives}

Whereas it is appreciated that the exact mechanism and theory of the aerodynamic lag is not fully understood, the experimental results from Pratt and Whitney for an NACA (2.1) 405 aerofoil operating into the separation region have been incorporated into the cascade theory developed in this present report and the solution for the damping derivatives found using the collocation method described earlier with three control points $\left(\frac{1}{4} c, \frac{1}{2} c, \frac{3}{4} c\right)$. The lag to be used at each of these stations was found from Pratt and Whit- 
TABLE II

Damping Derivative $m_{2}$ $M=0.5, \sigma=200^{\circ}$, zero stagger angle, Aerofoil section N.A.C.A. (2.1) 405

$\begin{array}{cccc}\text { Curve } & \text { Curves } & \text { Curve } & \text { Curve } \\ 6 & 7 \mathrm{a}, \mathrm{b} & \mathbf{8} & \mathbf{9}\end{array}$

\begin{tabular}{|c|c|c|c|c|c|c|c|}
\hline \multirow{2}{*}{$\begin{array}{l}\text { Reduced } \\
\text { frequency } \\
k\end{array}$} & \multicolumn{3}{|c|}{$\begin{array}{l}\text { Aerodynamic } \\
\text { 'Phase Lag' } \varphi\end{array}$} & \multirow{2}{*}{$\begin{array}{l}m_{2} \text { for } \\
s / c=0.75 \\
M=0.5\end{array}$} & \multirow{2}{*}{$\begin{array}{l}m_{2} \text { for } \\
s / c=1.00 \\
M=0.5\end{array}$} & \multirow{2}{*}{$\begin{array}{l}m_{2} \text { for } \\
\text { isolated } \\
\text { wing, } \\
\text { separation } \\
\text { from } \\
\text { leading } \\
\text { edge } \\
M=0\end{array}$} & \multirow{2}{*}{$\begin{array}{l}m_{2} \text { for } \\
\text { isolated } \\
\text { wing, } \\
\text { separation } \\
60 \% \\
\text { back from } \\
\text { leading } \\
\text { edge } \\
M=0\end{array}$} \\
\hline & $\frac{1}{4} c$ & $\frac{1}{2} c$ & $\frac{3}{4} c$ & & & & \\
\hline 0.02 & $\begin{array}{c}3^{\circ} 30^{\prime} \\
0\end{array}$ & $\begin{array}{l}3^{\circ} \\
0\end{array}$ & $\begin{array}{c}2^{\circ} 30^{\prime} \\
0\end{array}$ & $\begin{array}{c}-0.0218 \\
\text { Not calculated }\end{array}$ & $\begin{array}{l}-0.0220 \\
-0.0220\end{array}$ & -0.0094 & -0.0189 \\
\hline 0.10 & $\begin{array}{c}17^{\circ} \\
0\end{array}$ & $\begin{array}{c}16^{\circ} 30^{\prime} \\
0\end{array}$ & $\begin{array}{c}13^{\circ} \\
0\end{array}$ & $\begin{array}{c}-0.0812 \\
\text { Not calculated }\end{array}$ & $\begin{array}{l}-0.0998 \\
-0.0955\end{array}$ & -0.0498 & -0.0876 \\
\hline 0.20 & $\begin{array}{c}\mathbf{3 5}^{\circ} \\
0\end{array}$ & $\begin{array}{c}32^{\circ} 30^{\prime} \\
0\end{array}$ & $\begin{array}{c}25^{\circ} 30^{\prime} \\
0\end{array}$ & $\begin{array}{c}-0.1433 \\
\text { Not calculated }\end{array}$ & $\begin{array}{l}-0.1726 \\
-0.1593\end{array}$ & -0.0989 & -0.1800 \\
\hline 0.30 & $\begin{array}{c}52^{\circ} 30^{\prime} \\
0\end{array}$ & $\begin{array}{c}49^{\circ} \\
0\end{array}$ & $\begin{array}{c}38^{\circ} 30^{\prime} \\
0\end{array}$ & $\begin{array}{c}-0.1750 \\
\text { Not calculated }\end{array}$ & $\begin{array}{l}-0.2138 \\
-0.1989\end{array}$ & -0.1488 & -0.2724 \\
\hline 0.40 & $\begin{array}{c}70^{\circ} \\
0\end{array}$ & $\begin{array}{c}65^{\circ} 30^{\prime} \\
0\end{array}$ & $\begin{array}{c}51^{\circ} \\
0\end{array}$ & $\begin{array}{c}-\mathbf{0 . 1 6 9 3} \\
\text { Not calculated }\end{array}$ & $\begin{array}{r}-0.2126 \\
-0.2429\end{array}$ & -0.2085 & -0.3610 \\
\hline 0.50 & $\begin{array}{c}95^{\circ} \\
0\end{array}$ & $\begin{array}{c}81^{\circ} 30^{\prime} \\
0\end{array}$ & $\begin{array}{c}63^{\circ} 30^{\circ} \\
0\end{array}$ & $\begin{array}{c}-0.1488 \\
\text { Not calculated }\end{array}$ & $\begin{array}{l}-0.2498 \\
-0.2934\end{array}$ & -0.2486 & -0.4483 \\
\hline
\end{tabular}

Practical $\varphi$ values not available beyond $k=0.50$.

ney's results $(\varphi)$. The interblade phase angle $\sigma$ was taken to be $200^{\circ}$ after minimisation of critical flutter velocity with respect to $\sigma[9]$ and the results for the damping derivatives $l_{2}$ and $m_{2}$ as functions of reduced frequency for several gap-chord ratios are given in Figure 3 (Tables I, II). A Mach number of 0.5 was chosen for the cases presented and the $\varphi$ values are for an NACA (2.1) 405 series aerofoil operating near the stall. The situation when the aerodynamic lag in the separation region is not included is also shown for comparison and the loss of damping due to this aerodynamic lag can be seen as $k$ increases, especially for $l_{2}$. A typical numerical set of results for $l_{2}$ and $m_{2}$ is given in Tables I and II for a gap-chord ratio of unity.

As a further point of interest, several of Wood's results [5] for an oscillating single aerofoil with separation from a fixed point are also included for comparison, but it is pointed out that these latter results are for incompressible flow $(M=0)$.

The rather heavy computations were partly done at the Massachusetts Institute of Technology Computation Centre and the assistance of the staff 


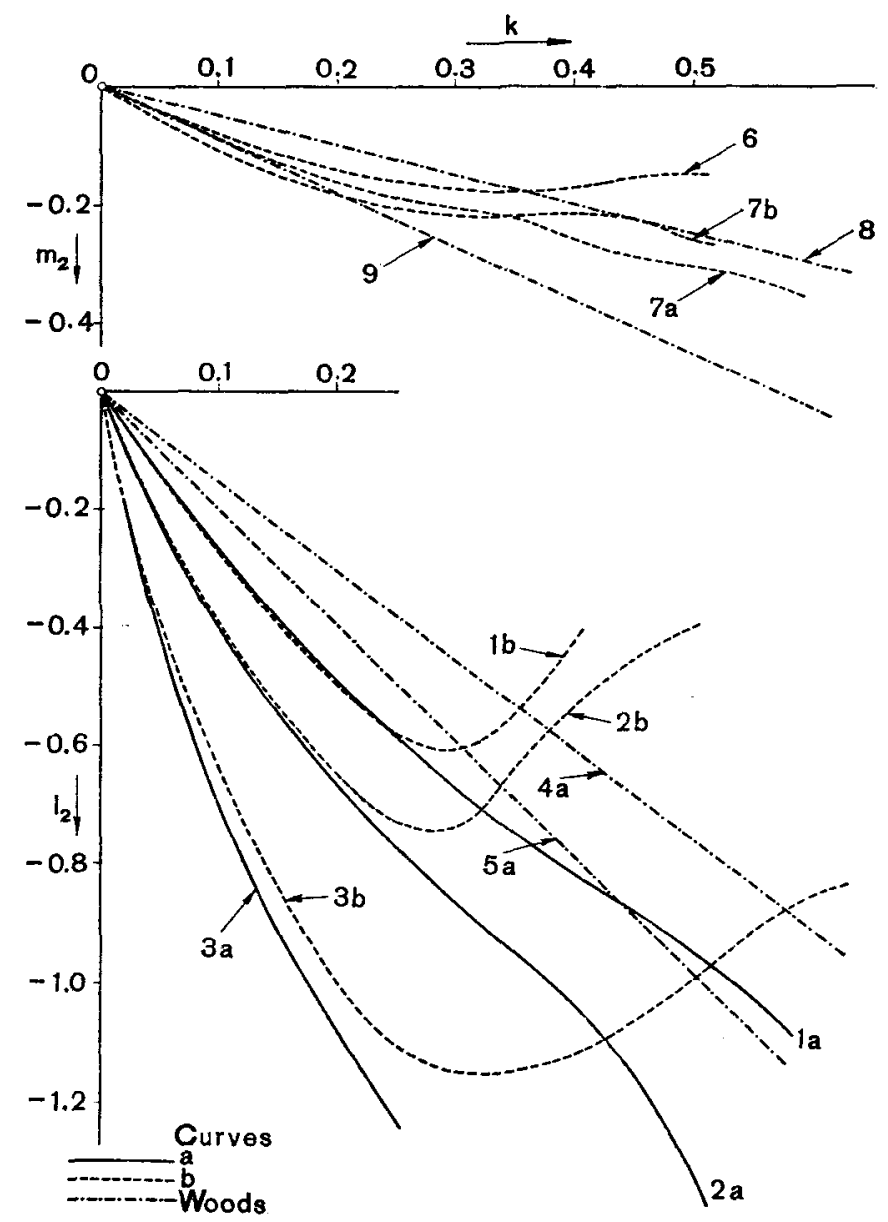

Figure 3. Damping Derivatives. Curves labelled as in Tables I, II Curves (a): without separation. Curves (b): with separation

is gratefully acknowledged. To obtain an accuracy of four decimal places for $A_{0}, A_{1}$, and $A_{2}$ the series $S_{1}$ and $S_{2}$ presented no fundamental problems - the only difficulties were with the series $S_{3}$ but these were more in the nature of patience and perseverance.

Distances were scaled with respect to unit semi-chord $b$ and sea-level conditions were used. Test amplitudes in the collocation process were taken in the range 0.1 to 0.2 of semi-chord, but the actual values chosen did not seem to affect the numerical results which were found to be sensibly independent of trial amplitudes. The interblade phase angle was taken as $200^{\circ}$ after a range of $\sigma$ values was considered for minimisation of critical flutter velocity with respect to $\sigma$. 


\section{Discussion}

The results for $M=0.5, \sigma=200^{\circ}$ show that there is a considerable loss of damping in all cases past $k \sim 0.25$ when separation is present. The damping is also reduced by decreasing the gap-chord ratio of the cascade. A very qualitative comparison of the results with those for an isolated aerofoil oscillating in incompressible flow with separation from a fixed arbitrary point [5] might suggest that damping is less for cases with separation from a fixed point than for periodic flow separation - for which the aerodynamic phase lag angles incorporated in the present numerical results apply.

When a fluttering blade moves into the separation region past the stall point the oscillations are those of a hysteretic system governed by a nonlinear differential-difference equation.

The stability of such blade oscillations in the presence of the upstream wakes has been studied separately [14] with a digital computer using the non-linear feedback analogue to the system and which has behaviour governed by the same equation. A dual input describing function [21] is applied to the analogous feedback system with consideration given to the aerodynamic phase lag and the program can include an initially small torsional motion. Recent computations have indicated from the resulting Nyquist diagrams that bounded regions for divergent oscillations can exist beyond $k \sim 0.25$ in most practical cases.

Finally, an interesting similarity is noted between the problem of a vibrating cascade of blades as studied herein and the vibrations of a flapping helicopter rotor blade for low inflow conditions. In a two dimensional model the spiral sheet of the wake shed below the vibrating rotor blade is approximated by a series of rows of linearly extended wakes appropriately phased. Both the hovering case [22] and that of slow forward motion require unsteady aerodynamic theory to obtain results in reasonable conformity with practice.

An approximate method of investigating the effects on the rotor blide flapping stability when the vibrating blade advances slowly has been presented [4] [23] and the results show that in similarity with the cascade problem, the damping and resonance conditions are directly related to the distribution of the previously shed rows of wakes below the blade - a result not obtainable from conventional flapping theory.

\section{Acknowledgement}

The author wishes to thank Dr. L. C. Woods, now at Balliol College, Oxford, for constructive advice on earlier work of the author on which this paper is based and Dr. F. O. Carta for his cooperation. 


\section{References}

[1] M. D. C. Doyle, An improved axial flow compressor design; J. Roy. Aero. Soc. 60 (1965), 791.

[2] L. C. Woods, On the theory of a cascade of stalled aerofoils; J. Australian Math. Soc. 1 part 2 (1960), 210.

[3] A. H. Low and L. C. Woods, Unsteady flow through a cascade of aerofoils; J. A ustralian Math. Soc. 1, part 2, (1960), 220.

[4] B. S. Thornton, Compressor blade flutter in jet engines; Ph. D. Thesis, School of Mathematics, University of New South Wales, 1960.

[5] L. C. Woods, Aerodynamic forces on an oscillating aerofoil fitted with a spoiler; Proc. Roy. Soc. A239 (1957) 328.

[6] G. M. Lilley, An investigation of the flexure-torsion flutter of aerofoils in cascade; College of Aeronautics, Cranfield, Report No 60 (1952).

[7] E. Reissner, Wind tunnel corrections for the two-dimensional theory of oscillating airfoils - Cornell Aeronautical Laboratory Report No SB-318-S-3 (1947)

[8] C. C. Chang \& V. C. Chu, Aerodynamic interference of cascade blades in synchronised oscillation; J. Appl. Mechanics (1955), 503.

[9] F. Lane, System mode shapes in the flutter of compressor blade rows; J. Aero. Sciences 23 (1956), 54.

[10] A. Mendelson and R. W. Carroll, Lift and moment equations for oscillating airfoils in an infinite unstaggered cascade; N.A.C.A. Tech. Note 3263 July 1954.

[11] H. von Söhngen and E. Meister, Beitrag zur Aerodynamik eines schwingenden Gitters; Zeit. f. Ang. Math. u. Mech., 38 (1958), 442.

[12] J. E. McCune, A three dimensional theory of axial flow compressor blade rows; $J$. Aero/Space Sciences, 25 (1958), 544.

[13] C. Possio, L'azionne aerodynamica sul profilo, oscillanto in un fluido compressibile a velocita iposonora; Aerotecnica, Roma 18 (1938), 441.

[14] T. M. Park and B. S. Thornton, Stability of non-linear difference-differential equations in aerodynamics; Proc. Australian Computer Conference, May 1960, paper B19.2.

[15] G. N. Watson, Theory of Bessel functions, Cambridge Univ. Press (1922).

[16] L. Infield, V. G. Smith and W. Z. Chien, On some series of Bessel functions; J. Math. \& Physics, 26 (1947) 22.

[17] E. C. Titchmarsh, Theory of functions, Clarendon Press, Oxford (1948).

[18] H. Runyan and D. S. Woolston, Some considerations on the air forces on a wing oscillating between two walls for subsonic compressible flow; J. Aero. Sciences $22(1955), 41$.

[19] Y. L. Luke, Computation of oscillatory integrals of the type $\int_{a}^{b} f(y) e^{i \lambda y} d y$. Proc. Cambridge Phil. Soc. 50, part 2 (1954), 269.

[20] F. Lane and M. Friedman, Theoretical investigation of subsonic oscillatory blade-row aerodynamics; N.A.C.A. Tech. Note 4136 (1958).

[21] J. C. West, J. L. Douce and R. K. Livesley, The dual input describing function and its use in the analysis of non-linear systems; Proc. Inst. Electrical Engineers Paper No. $1877 \mathrm{M}(1955), 463$.

[22] R. G. Loewy, A two-dimensional approximation to unsteady aerodynamics of a rotary wing; Cornell Aeronautical Lab. Report No. 75 (1955).

[23] W. T. F. Lau, Master of Engineering Thesis, School of Mechanical Engineering, University of New South Wales, 1961.

Honeywell E. D. P. Division

55 Macquarie St, Sydney 